\title{
Ribosome profiling reveals sequence-independent post-initiation pausing as a signature of translation
}

\author{
Yan $\operatorname{Han}^{1,2, *}$, Xiangwei Gao ${ }^{1, *}$, Botao Liu ${ }^{3}$, Ji Wan $^{1}$, Xingqian Zhang ${ }^{1}$, Shu-Bing Qian ${ }^{1,3}$ \\ ${ }^{1}$ Division of Nutritional Sciences, Cornell University, Ithaca, NY 14853, USA; ${ }^{2}$ Department of Infectious Diseases, Ruijin Hospital, \\ Shanghai Jiaotong University School of Medicine, Shanghai 200025, China; ${ }^{3}$ Graduate Field of Genetics, Genomics and Develop- \\ ment, Cornell University, Ithaca, NY 14853, USA
}

The journey of a newly synthesized polypeptide starts in the peptidyltransferase center of the ribosome, from where it traverses the exit tunnel. The interior of the ribosome exit tunnel is neither straight nor smooth. How the ribosome dynamics in vivo is influenced by the exit tunnel is poorly understood. Genome-wide ribosome profiling in mammalian cells reveals elevated ribosome density at the start codon and surprisingly the downstream 5th codon position as well. We found that the highly focused ribosomal pausing shortly after initiation is attributed to the geometry of the exit tunnel, as deletion of the loop region from ribosome protein L4 diminishes translational pausing at the 5th codon position. Unexpectedly, the ribosome variant undergoes translational abandonment shortly after initiation, suggesting that there exists an obligatory step between initiation and elongation commitment. We propose that the post-initiation pausing of ribosomes represents an inherent signature of the translation machinery to ensure productive translation.

Keywords: ribosome; translation

Cell Research (2014) 24:842-851. doi:10.1038/cr.2014.74; published online 6 June 2014

\section{Introduction}

Ribosomes decode the genetic information in mRNA and convert it into the amino acid sequences of proteins. During mRNA translation, the ribosome does not proceed at a constant rate but rather in a stop-and-go movement manner [1]. Variations of elongation speed may result from local stable mRNA structures [2], the presence of rare codons [3], or the interactions between the nascent chain and the ribosome and/or exogenous factors [4]. Frequent translational pausing may have functional importance for the cellular production line. For instance, ribosome stalling could trigger frame-shifting or translational abandonment $[5,6]$. A growing body of evidence suggests that cells purposely employ ribosomal pausing

*These two authors contributed equally to this work.

Correspondence: Shu-Bing Qian

E-mail: sq38@cornell.edu

Received 12 February 2014; revised 30 March 2014; accepted 24 April

2014; published online 6 June 2014 as a regulatory mechanism to maintain intracellular protein homeostasis. For example, translational delays in specific regions facilitate co-translational folding of nascent chains [7]. Ribosomes also adopt early elongation pausing in response to various stress conditions $[8,9]$. Given the wide range of impacts of ribosome pausing on cellular systems, a fundamental question is to dissect the mechanistic basis underlying translational pausing and determine the physiological consequences when ribosomes stall on individual mRNAs.

Despite several decades since the first description of ribosome pausing and stacking during translation [10], it was not possible until now to measure ribosome dynamics in vivo at the genome-wide scale. Ribosome profiling or Ribo-seq, based on deep sequencing of ribosome-protected mRNA fragments (RPFs), has proven to be powerful in determining ribosome positions and densities across the entire transcriptome [11]. Many research groups have successfully applied Ribo-seq to a wide range of organismal species and reported pervasive pause sites along the coding region (CDS) of mRNAs [12-18]. From bacteria to human cells, the most consistent pause 
sites are located at the start and stop codons, which is in line with the notion that ribosomes tend to pause during initiation and termination [10]. Ribosome profiling also captures many sequence-dependent stalling sites [15]. However, much less is known about translational pausing governed by factors other than cis-elements.

Here, we report and characterize a specific ribosomal pausing at the 5th codon position of mRNA CDSs in mammalian cells. Unlike sequence-dependent elongation pausing, the ribosome stalling shortly after initiation is attributed to the geometry of the exit tunnel. Importantly, modifying the exit tunnel not only reduces the ribosomal pausing at the 5th codon position, but results in an unexpected translational abandonment. Our finding suggests a rich communication between the nascent chain and the translation machinery in regulating ribosome kinetics during translation.

\section{Results}

Ribosome profiling reveals post-initiation pausing

We applied a modified Ribo-seq to HEK293 cells and examined the average pattern of ribosome density across the entire transcriptome $[8,19]$. As expected, the mapped RPF reads start an abrupt accumulation at the annotated start codon [11] (Figure 1A). Interestingly, we observed another prominent peak at the $12 \mathrm{nt}$ downstream position, corresponding to the 5th codon position of the CDS (Figure 1A, red arrow). This is not due to cycloheximide (CHX)-mediated translational arrest because the same feature is maintained in cells without any drug treatment (Figure 1A, left panel). The ribosomal pausing at the
A

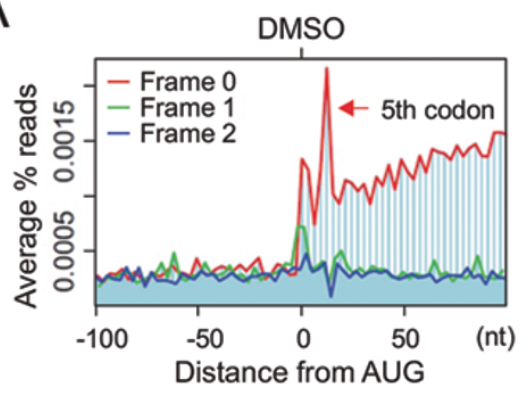

C
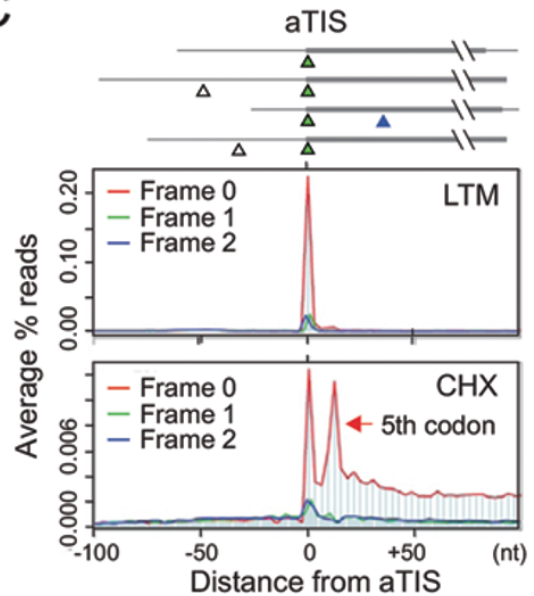
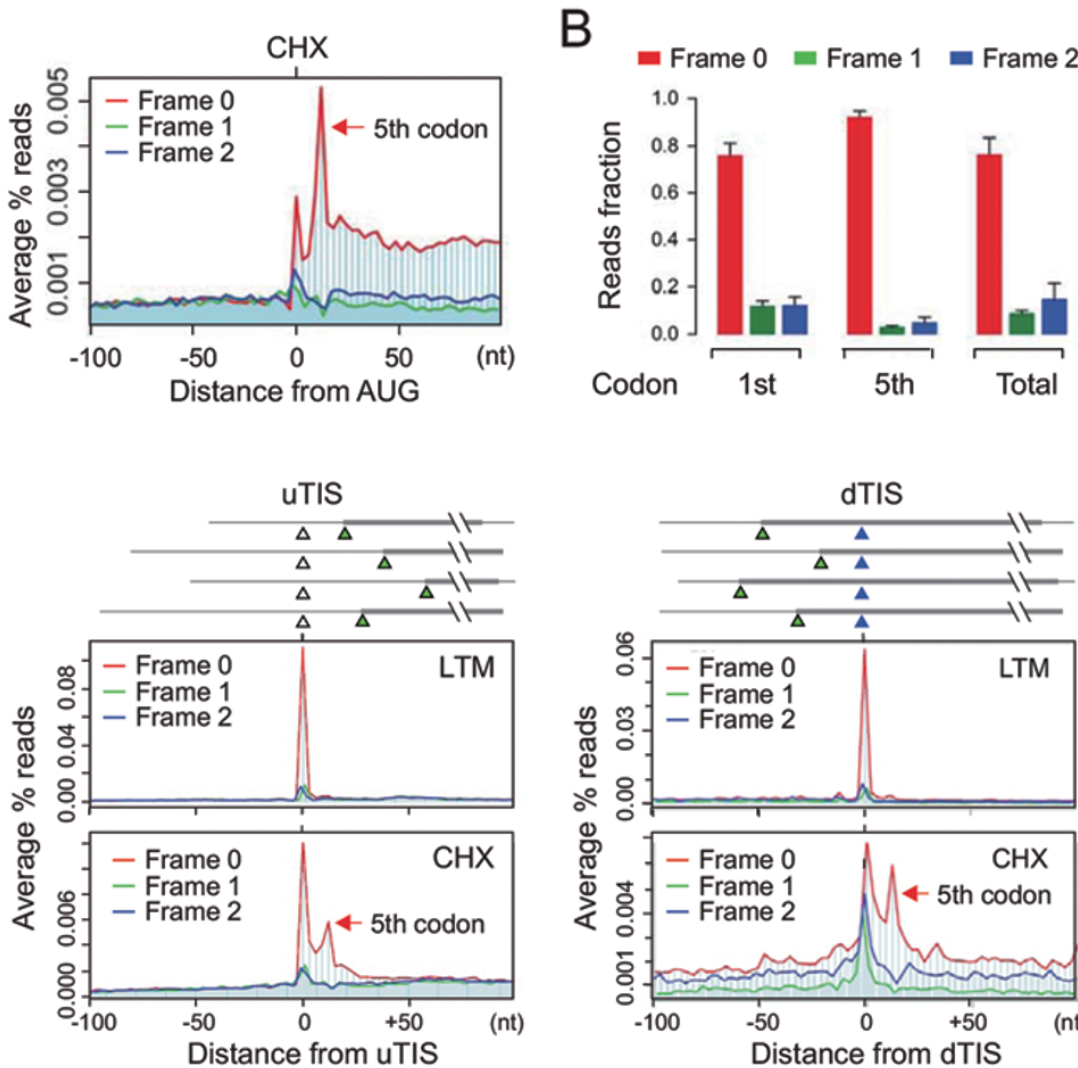

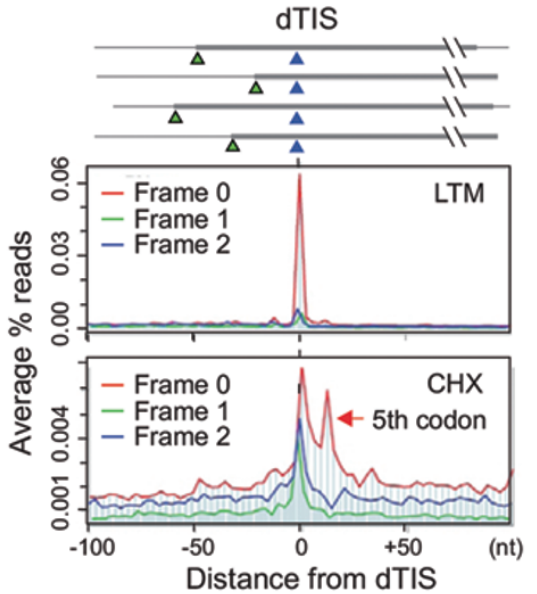

Figure 1 Characterization of post-initiation pausing. (A) Metagene analysis of RPFs obtained from HEK293 cells treated with CHX (right) or DMSO control (left). All mapped reads are aligned at the annotated start codon AUG and stratified by frames. The read density at each nucleotide position is averaged using the P-site of RPFs. The red arrow indicates the 5th codon position. (B) Read fractions relative to the annotated reading frame are calculated for RPFs mapped to the 1st (AUG) codon, 5th codon, or the entire CDS. Data are shown as means $\pm \mathrm{SD}, n=4$. (C) Metagene analysis of RPFs obtained from HEK293 cells treated with LTM (upper) or CHX (bottom). All mapped reads are aligned at the annotated start codon (aTIS, green triangle; left panel), upstream TIS (UTIS, white triangle; middle panel), or downstream TIS (dTIS, blue triangle; right panel). The read density at each nucleotide position is averaged using the P-site of RPFs. The red arrow indicates the 5th codon position. 
5th codon position is highly reproducible as evidenced by the similar pattern of RPF distribution in a total of 4 independent biological replicates (Supplementary information, Figure S1). To confirm whether the same feature also exists in different cell types, we conducted Ribo-seq in HeLa cells and a mouse embryonic fibroblast (MEF) cell line. Both cells showed consistent pattern of RPFs with prominent peaks of ribosome density at both the start codon and the 5th codon position (Supplementary information, Figure S2). Thus, the excess of ribosome density at the 5 th codon position represents a strong ribosomal pausing shortly after initiation.

The post-initiation pausing bears several unique features that distinguish it from the typical ribosome pausing at the start codon. First, the RPFs recovered at the 5th codon position demonstrate a near perfect framing or phasing with $>90 \%$ of reads mapped to the same frame as the annotated ORFs (Figure 1A and 1B). This is in contrast to the RPF reads derived from other CDS regions including the AUG start codon, in which about a quarter of the reads are out of the annotated reading frame (Figure 1B). Second, RPFs derived from the 5 th codon position have different size distribution in length (Supplementary information, Figure S3). While the majority of footprints mapped to the entire CDS were 29mer, a substantial proportion of RPFs mapped to the 5th codon position are $27-$ mer. These results suggest that the stalled ribosome at the 5 th codon position might adopt a more compact conformation than the others. Supporting this notion, recent studies demonstrated that the length of footprints could be influenced by ribosome dynamics $[20$, 21].

To rule out the possibility that the prominent ribosomal pausing at the 5th codon position is due to reinitiation, we segregated initiating ribosomes from elongating ribosomes by applying a translation inhibitor lactimidomycin (LTM). Unlike CHX that freezes all the ribosomes engaged on mRNAs, LTM preferentially acts on the initiating ribosome but not the elongating one [22]. As we reported previously, LTM-based ribosome profiling (GTI-seq) offers global mapping of translation initiation sites (TIS) at single-nucleotide resolution [19]. GTIseq revealed a pronounced single peak highly focused at the annotated TIS with few reads recovered in the downstream region (Figure 1C, left panel). The lack of LTM-associated RPF reads at the 5th codon position strongly indicates that the ribosomal pausing at this position is a true elongation event. GTI-seq also permits identification of alternative start codons, including upstream TIS and downstream TIS sites [19]. To examine whether alternative initiation is also followed by downstream ribosomal pausing, we aligned transcripts by the alternative TIS sites identified by GTI-seq (Figure 1C, middle and right panels). Remarkably, the CHX-associated RPFs exhibit dominant peaks not only at the corresponding TIS positions, but also at the downstream 5th codon position. Thus, post-initiation pausing represents a universal feature for ribosomes after initiation from both the annotated and the alternative start codons.

When the ribosomal P-site is mapped at the 5th codon position, its footprint coincidently starts with AUG at the $5^{\prime}$ end. One possibility is that these RPFs are preferentially obtained after RNase I digestion or selectively amplified during deep sequencing. However, this is not the case. The frequency of RPFs with AUG at the $5^{\prime}$ end is among the average compared to RPFs starting with other sequences (Supplementary information, Figure S4). Furthermore, many internal AUG codons are not associated with the downstream pausing. Therefore, the elevated ribosome density at the 5 th codon position after the annotated start codon is likely coupled with the initiation event.

\section{Characterization of post-initiation pausing}

To investigate possible mechanisms underlying post-initiation pausing at the 5th codon position, we first examined whether ribosome stalling at this specific position is sequence dependent. However, we found a similar codon composition at the 5 th codon position to the one averaged across the entire CDS (Supplementary information, Figure S5). To find possible general relationships between the functions of genes and post-initiation pausing, we searched for common biological themes in transcripts with high and low ribosomal pausing at the 5th codon position. Gene ontology term analysis revealed no specific gene functions associated with either strong or weak post-initiation pausing (Supplementary information, Figure S6). These results indicate that the ribosomal pausing shortly after initiation is sequence independent, likely representing a translational event inherently associated with the translation machinery per se.

We next asked whether ribosomal pausing at the 5th codon position is a result of ribosomal stacking during elongation. To this end, we isolated the monosome fraction followed by Ribo-seq (Supplementary information, Figure S7). Since every transcript in the monosome has only one ribosome, the ribosome behavior is not influenced by any neighboring ribosomes. Despite much less RPF density in the CDS region, the monosome fraction maintained prominent peaks at both the start codon and the 5th codon position (Supplementary information, Figure S7). This result further supports the notion that post-initiation pausing reflects an intrinsic feature of ribosomes shortly after initiation. 
Having characterized the post-initiation pausing in mammalian cells, we attempted to expand our findings to other species whose Ribo-seq data are available in the literature. Reanalyzing the RPF reads using the same pipeline revealed similar post-initiation pausing in other eukaryotic cells (Supplementary information, Figure S8), including budding yeast $S$. cerevisiae [11], nematode $C$. elegans [17], and zebrafish D. rerio [16]. Interestingly, the most recent report of mitochondrial ribosome profiling also showed similar ribosomal pausing at the 5th codon position [23]. Notably, the relative extent of post-initiation pausing varies among different studies and we cannot exclude the possibility that technical differences (e.g., $\mathrm{Mg}^{2+}$ concentration in polysome buffer) may cause variations of ribosome density at the 5 th codon position. Considering its omnipresence in multiple species, it is likely that the post-initiation pausing is a general feature for different types of translation machinery.

\section{Post-initiation pausing is attributed to the geometry of the exit tunnel}

Given the sequence-independent but position-specific feature, the observed post-initiation pausing is likely governed by the ribosome exit tunnel. The interior of the ribosome exit tunnel is neither straight nor smooth with an evident constriction site formed by RPL4 and RPL17 [24] (Figure 2A). To investigate whether the highly focused ribosomal pausing shortly after initiation is attributed to the geometry of the exit tunnel, we focused on RPL4 because it is the first ribosomal protein that the nascent chain potentially interacts within the exit tunnel. We constructed a RPL4 mutant by deleting the most conserved HRSG loop region [25] (Supplementary information, Figure S9). After overexpression in HEK293 cells, myc-tagged RPL4( $\Delta$ loop) mutant showed a similar cellular localization to the wild type (Figure 2B). In addition to the nucleolus localization that is typical for ribosome subunit assembly, both the wild-type and the mutant RPL4 showed the same cytoplasmic distribution as ribosomal P0, a protein associated only with the assembled ribosome complex. To confirm that RPL4( $\Delta$ loop) is able to incorporate into the assembled $80 \mathrm{~S}$ ribosome complex in the cytoplasm, we performed immunoprecipitation (IP) assay using an anti-myc antibody in HEK293 cells stably expressing wild-type RPL4 or RPL4( $\Delta$ loop). The presence of a myc tag at the $\mathrm{C}$ terminus of the transgene prevents precipitation of ribosomes synthesizing the RPL4 polypeptide itself. In addition, we converted the polysome into monosome by RNase I digestion prior to anti-myc IP to exclude indirect ribosome pull-down (Figure $2 \mathrm{C}$ ). Both small and large ribosomal proteins were readily precipitated by anti-myc antibody from cells ex-
A
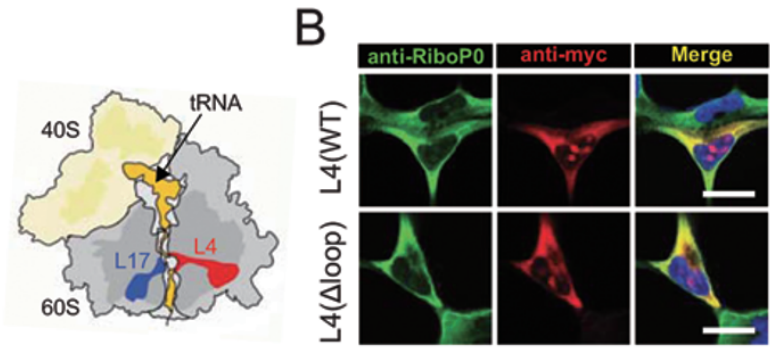

C
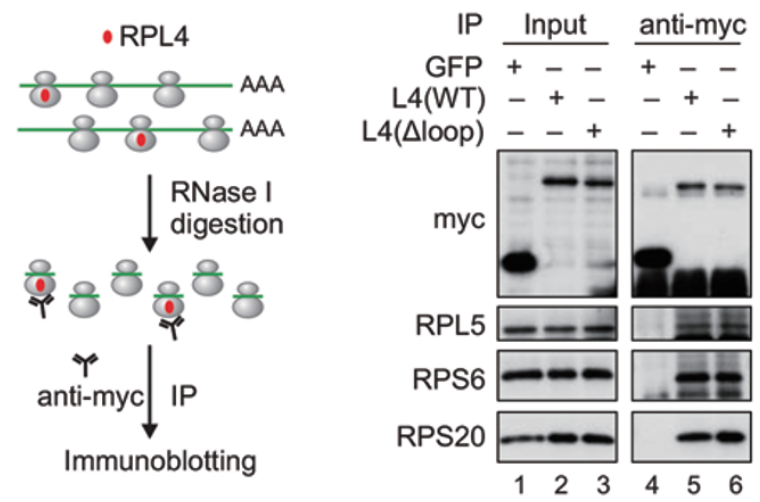

$\mathrm{D}$

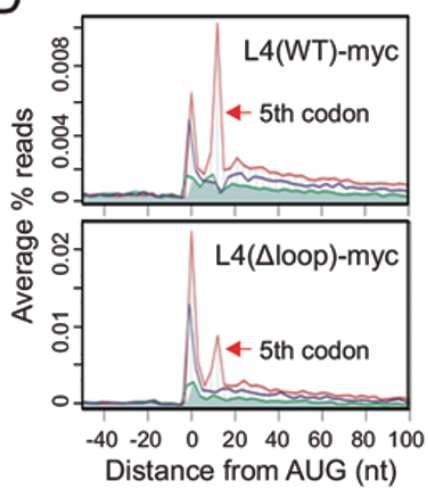

E

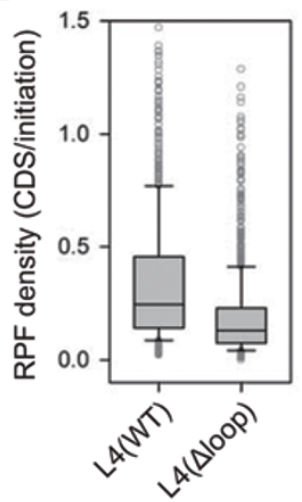

Figure 2 Reduced post-initiation pausing for ribosomes bearing L4 mutant. (A) Schematic structure of ribosomes highlighting the exit tunnel. tRNA and the nascent chain are shown in orange, RPL4 in red, and RPL17 in blue. (B) Cellular localization of L4 wild type and $\Delta$ loop mutant is examined in HEK293 cells using immunostaining. Scale bar, $10 \mu \mathrm{m}$. (C) Myc-tagged L4 is incorporated into ribosome complexes. Polysome fractions from transfected HEK293 cells were treated with RNase I prior to anti-myc IP. Both the input and the immunoprecipitates were blotted using antibodies as indicated. (D) Metagene analysis of RPFs obtained from purified ribosomes bearing L4 wild type (top) and $\Delta$ loop mutant (bottom). All mapped reads are aligned at the annotated start codon AUG and stratified by frames. (E) Read density over CDS relative to the initiation site (1st - 5th) is shown in box plots.

pressing either wild-type RPL4 or RPL4( $\triangle$ loop) (Figure $2 \mathrm{C}$, lanes 5 and 6 ), but not GFP control. Thus, deleting 
the loop region does not prevent RPL4 from integrating into the assembled $80 \mathrm{~S}$ ribosome complexes.

We next examined the behavior of ribosomes bearing myc-tagged RPL4 by Ribo-seq of transfected cells. To exclude the RPFs derived from ribosomes containing endogenous RPL4, we conducted selective ribosome profiling by purifying myc-tagged ribosomes after RNase I digestion. Thus, all the obtained RPFs are exclusively derived from ribosomes bearing myc-tagged RPL4. As expected, ribosomes containing RPL4(WT) showed elevated ribosome density at both the start codon and the 5th codon positions (Figure 2D). However, RPL4( $\Delta$ loop)-incorporated ribosomes exhibited a substantial reduction of ribosome density at the 5 th codon position relative to the start codon (Figure 2D, bottom panel). The diminished post-initiation pausing is also evident on individual transcripts, including the relatively long $P 4 H B$ and the short RPL23 (Supplementary information, Figure S10). Notably, the ribosome density at the start codon remains high for ribosomes containing RPL4( $\Delta$ loop), strongly indicating that the assembly of $80 \mathrm{~S}$ ribosomes at the start codon is not affected by this ribosome variant.

\section{Ribosomes with altered exit tunnel lead to abortive trans- lation}

For ribosomes with modified exit tunnel, the reduced post-initiation pausing is expected to promote the rate of translation. Yet the RPL4( $\Delta$ loop)-associated RPFs showed an obvious decrease of footprint density over the downstream CDS region relative to the start codon (Figure 2E). This surprising finding suggests that the ribosome variant with a modified exit tunnel does not support continuous translation elongation. This could be explained by ribosome drop-off that occurs in the presence of RPL4( $\Delta$ loop). To test this possibility, we inspected the distribution of myc-tagged RPL4 in ribosome fractions resolved by sucrose gradient velocity sedimentation (Figure 3A). Myc-tagged RPL4(WT) completely behaved like the endogenous one as evidenced by their nearly identical distribution in polysome fractions. RPL4( $\Delta$ loop), in contrast, showed an evident decrease in the polysome fractions relative to the endogenous RPL4 despite its comparable enrichment in the monosome (Figure 3A and 3B). These results suggest that an altered ribosomal exit tunnel may cause ribosome dissociation shortly after initiation.

Notably, the distance between the peptidyltransferase center (PTC) and the RPL4 loop region is more than five amino acids [24]. Removing the RPL4 loop region does not seem to clog or close the exit tunnel as we observed no additional pausing sites within the 30 -codon window for ribosomes bearing RPL4( $\Delta$ loop) (Figure 2D). To ex- amine whether translational abandonment mainly occurs before the nascent chain emerges out of the ribosome, we performed nascent chain IP in HEK293 cells expressing a GFP reporter with an N-terminal Flag-tag [8]. Early ribosome dissociation would prevent anti-Flag antibody from precipitating translating ribosomes. For cells expressing RPL4(WT), anti-Flag IP could pull down considerable amount of myc-tagged RPL4 along with other ribosome proteins (Figure 3C, lane 5). However, in cells expressing RPL4( $\Delta$ loop), very little RPL4( $\Delta$ loop) was co-precipitated by anti-Flag antibody when compared to other ribosome proteins (Figure 3C, lane 6). This result is consistent with early abandonment of translation for ribosomes with modified exit tunnel.

For ribosomes with altered exit tunnel, it is unclear which event happens first: reduced post-initiation pausing or ribosome dissociation. Although the lack of post-initiation pausing could trigger ribosome drop-off, it is equally possible that the diminished post-initiation pausing is a direct consequence of abolished translation shortly after initiation. In the latter scenario, dissociation of ribosomes bearing RPL4( $\Delta$ loop) must occur before the nascent chain reaches the 5 th codon position. Despite lots of efforts, we were unable to determine the length of tRNA-linked short peptides associated with ribosomes bearing RPL4( $\Delta$ loop). Nevertheless, our results argue that the very first few amino acids are able to sense the integrity of the ribosomal exit tunnel in an active manner and post-initiation pausing of ribosomes may facilitate elongation commitment.

\section{Ribosomes with altered exit tunnel lead to cellular toxicity}

The frequent translational abandonment is expected to be detrimental to cellular functions. This was indeed the case. We examined cell viability after knocking down the endogenous RPL4 using lentiviruses expressing small hairpin RNA (shRNA) targeting the 3' UTR of RPL4. Consistent with the essential role of RPL4 in protein production, we observed progressive cellular toxicity in control cells expressing GFP after RPL4 knockdown (Figure 4A). Only cells expressing wild-type RPL4, but not RPL4(loop), were able to maintain higher cell viability. Thus, the post-initiation pausing governed by the ribosomal exit tunnel represents an obligatory step between initiation and elongation.

\section{Discussion}

A longstanding question in translational control is to systemically understand physiological purposes of ribosomal pausing. mRNA structure, tRNA abundance, and amino acid sequence all have the potential to impact the 
A
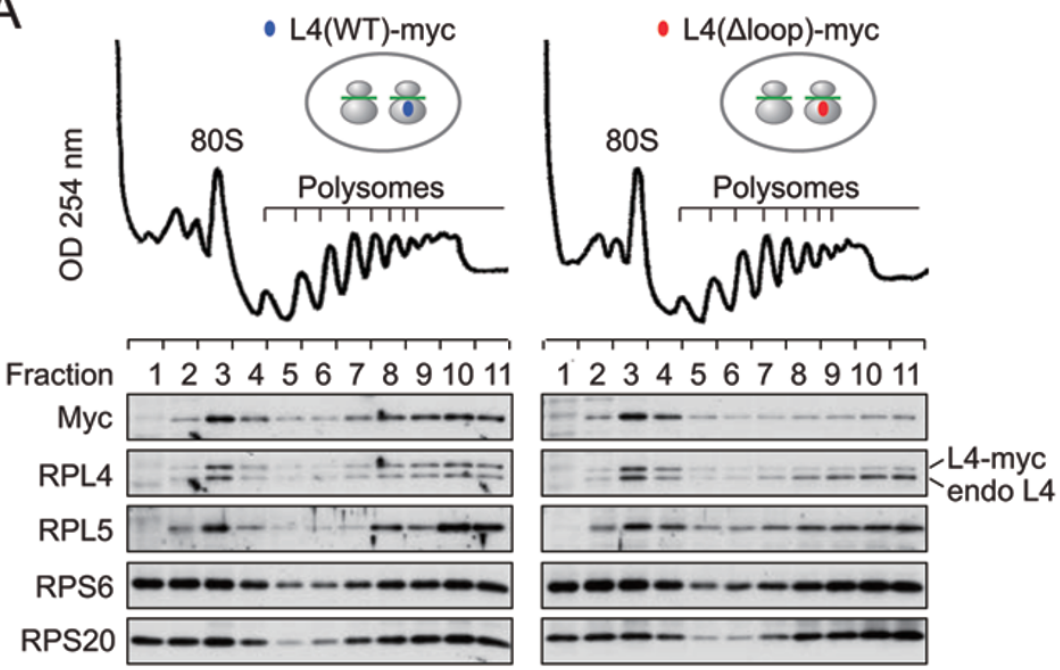

B
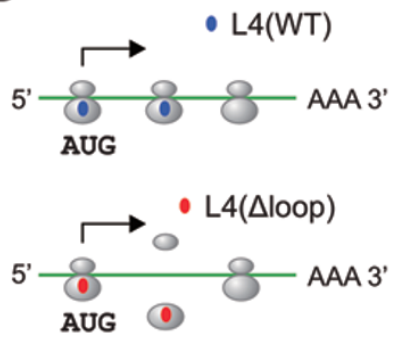

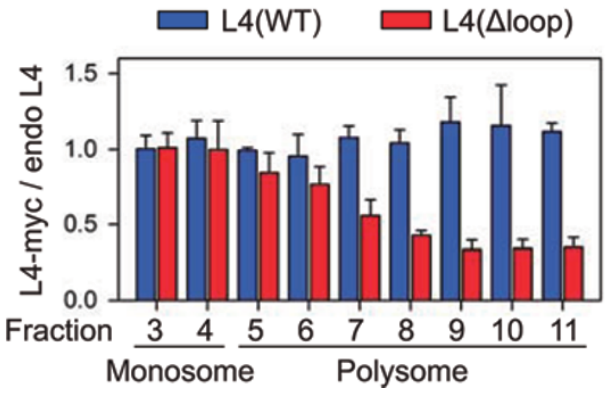

C

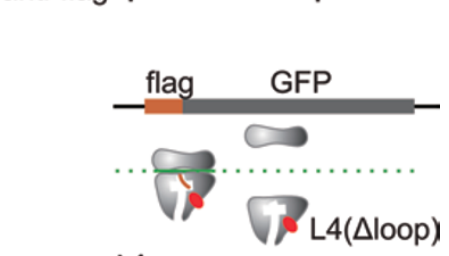

anti-flag $\Upsilon$

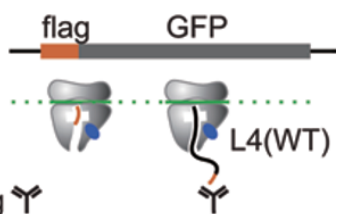

GFP $\frac{\text { Input }}{+--} \frac{\text { anti-flag IP }}{+--}$

$\mathrm{L} 4(\mathrm{WT})-+-\ldots+$

$\mathrm{L} 4(\Delta \mathrm{loop})-{ }_{-}+-+$

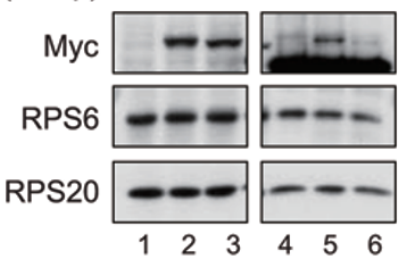

Figure 3 Ribosomes bearing L4 mutant lead to abortive translation. (A) Polysome profiles of HEK293 cells expressing L4 wild type or loop mutant. The bottom panel shows the distribution of exogenous and endogenous ribosome proteins in ribosome fractions. (B) A simple model of translational abandonment for ribosomes bearing $L 4(\Delta$ loop) mutant. The right panel shows the quantification of myc-tagged L4 relative to the endogenous L4 in ribosome fractions as shown in A. Data are shown as means $\pm \mathrm{SD}, n=3$. (C) Schematic for nascent chain IP assay using a HEK293 cell line expressing flag-GFP (top panel). Transfected HEK293 cells were lysed and treated with RNase I prior to anti-flag IP. Both the input and the immunoprecipitates were blotted for ribosomal proteins as indicated (bottom panel).

ribosome dynamics. Based on multiple empirical data sets, here we report a sequence-independent but position-specific ribosomal pausing governed by the geometry of the exit tunnel. Several lines of evidence support the notion that the ribosome pausing at the 5th codon position serves as a functional signature of translation. First, post-initiation pausing of ribosomes is nearly a universal feature in a wide range of organisms. With an ever growing application of ribosome profiling in different cell types and tissues, we expect to see a more comprehensive picture illuminating ribosome dynamics between initiation and elongation. Second, we provide direct evidence that modifying the exit tunnel influences the extent of ribosomal pausing shortly after initiation. To our knowledge, this is the first example of ribosomal pausing associated with the translation machinery. Third, post-ini- tiation pausing of ribosomes is of physiological significance as elimination of post-initiation pausing is coupled with translational abandonment. Together, these results argue that the ribosome behavior is intimately controlled via the constant interaction between the growing nascent chain and the translation machinery (Figure 4B).

It has long been believed that, after the assembly of $80 \mathrm{~S}$ ribosome at the start codon, elongation ensues via a simple repetitive process comprising ribosomal translocation and tRNA recycling. However, a growing body of evidence suggests that the synthesis of the very first 4 amino acids possesses special features. In prokaryotes, the presence of macrolides allows synthesis of at least 4 amino acids before peptidyl-tRNA release even though the macrolide binding sites is far below the PTC [26]. It has also been reported that overexpression of very short 
A

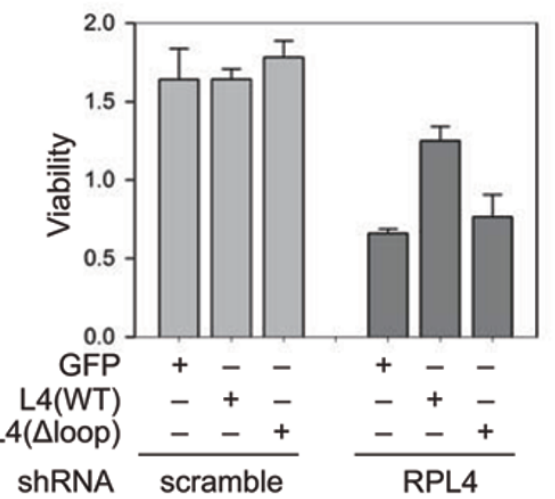

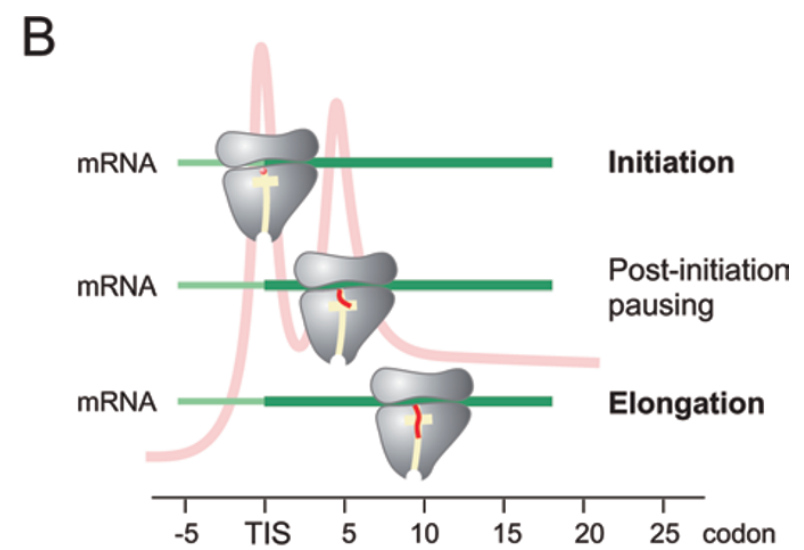

Figure 4 Physiological significance of post-initiation pausing. (A) HEK293 cells expressing GFP control, L4 wild type, or $\Delta$ loop mutant were infected with lentiviruses encoding shRNA targeting endogenous L4 or scramble control. Cell viability was measured and quantified. Data are shown as means $\pm \mathrm{SD}, n=3$. (B) A model for post-initiation pausing of ribosomes. After initiation, the nascent chain (red) traverses the exit tunnel (yellow). At the 5th codon position, the paused ribosome undergoes conformational changes, thereby facilitating elongation commitment by preventing nascent chain from off-route and abortive translation.

minigenes triggers ribosome drop-off. However, when the ORF length increased from three codons to seven, the drop-off frequency decreased significantly [27]. These findings can be explained by the existence of an additional step between initiation and elongation. It will be interesting to use single molecule fluorescence approach to elucidate ribosome dynamics in real time during the first several cycles of elongation [28].

The mandatory pausing of ribosomes at the 5th codon position is advantageous to ensure elongation commitment. The exit tunnel is the preferred but perhaps not the only pathway from the PTC. Some experimental results suggest that the nascent chain could leave the PTC along other routes [29]. We surmise that the post-initiation pausing of ribosomes allows the very first few amino acids to find the correct exit route. This unique interaction by the first amino acids could potentially trigger local conformational change of ribosomes before entering the exit tunnel. Interestingly, recent cryoelectron microscopy of eukaryotic ribosomes revealed discontinuous electron density of nascent chains after $\sim 4$ amino acids below the PTC [24], a strong indication of ribosome plasticity. In addition to RPL4, nascent chains undergo extensive interaction with many other ribosomal proteins, such as RPL17 and RPL39. Biochemical evidence suggested that the tunnel topology must be flexible to accommodate the nascent chain [30]. Given the unique feature of stalled ribosomes at the 5th codon position, post-initiation pausing of ribosomes represents a physiological signature of the translation machinery to ensure productive translation.

\section{Materials and Methods}

\section{Cells and reagents}

HEK293, HeLa, and MEF cells were maintained in Dulbecco's Modified Eagle's Medium (DMEM) with 10\% fetal bovine serum (FBS). CHX, puromycin, and erythromycin were purchased from Sigma. LTM was provided by Ben Shen (Scripps Florida) as described previously [19]. Anti-RPS6 antibody was purchased from Cell Signaling Technology; anti-myc from Santa Cruz Biotechnology; a human polyclonal autoimmune antisera targeting ribosomal P is from Immunovision; anti-flag, anti-RPS20, and anti-RPL5 antibodies from Sigma. Plasmid transfection was performed using Lipofectamine 2000 (Invitrogen) following the manufacturer's instruction.

\section{Plasmids and lentiviral shRNAs}

The full-length RPL4 gene was amplified by PCR followed by direct cloning into pcDNA3.1-myc-His vector (Invitrogen). To construct the RPL4( $\triangle$ loop) mutant, RPL4 fragments corresponding to CDS 1-252 nt and 262-1 $281 \mathrm{nt}$ were amplified by PCR followed by ligation using a BamHI restriction site. RPL4 shRNA targeting sequences (human: 5'-TGAATAAAGACCTGATTATAC-3') and control sequence (5'-AACAGTCGCGTTTGCGACTGG-3') were designed based on RNAi consortium at Broad Institute (http://www.broad.mit.edu/rnai/trc). All shRNA targeting sequences were cloned into DECIPHER ${ }^{\mathrm{TM}}$ pRSI9-U6-(sh)-UbiCTagRFP-2A-Puro (CELLECTA). Lentiviral particles were packaged using Lenti-X 293T cells (Clontech). Virus-containing supernatants were collected at $48 \mathrm{~h}$ after transfection and target cells were infected in the presence of $8 \mu \mathrm{g} / \mathrm{ml}$ polybrene.

\section{Immunofluorescence staining}

Cells grown on glass coverslips were fixed in $4 \%$ paraformaldehyde and permeabilized by $0.2 \%$ Triton X-100. After blocking 
in $2 \%$ BSA in PBS, fixed cells were incubated with the primary antibody at $4{ }^{\circ} \mathrm{C}$ overnight followed by 1 -h incubation at room temperature with Alexa Fluor-labeled secondary antibodies. Cells were then washed with PBS and incubated for 5 min in PBS supplemented with Hoechst to counter-stain the nuclei. After a final wash with PBS, coverslips were mounted onto slides and viewed using a confocal microscope (Zeiss LSM710).

\section{Immunoprecipitation}

Cells were harvested in ice-cold lysis buffer (10 mM Hepes, pH 7.4, $100 \mathrm{mM} \mathrm{KCl}, 5 \mathrm{mM} \mathrm{MgCl}, 100 \mu \mathrm{g} / \mathrm{ml} \mathrm{CHX)} \mathrm{and} \mathrm{lysed}$ with Lysing Matrix D (Fisher). After centrifugation at $4{ }^{\circ} \mathrm{C}$ and $10000 \times g$ for $10 \mathrm{~min}$, supernatant was collected, and treated with RNase I (Ambion) at $4{ }^{\circ} \mathrm{C}$ for $1 \mathrm{~h}$. Digestion was neutralized with SUPERase inhibitor (SUPERase_In, Ambion) and the samples were incubated with anti-myc beads (Sigma) or anti-flag beads (Sigma) at $4{ }^{\circ} \mathrm{C}$ overnight. Immunoprecipitates were washed 3 times with lysis buffer. The washed beads were resuspended in $1 \times$ SDS sample buffer (100 mM Tris, pH 6.8, 2\% SDS, 15\% glycerol, $5 \% \beta$-mercaptoethanol, $0.1 \%$ bromophenol blue), boiled for $5 \mathrm{~min}$, and analyzed by immunoblotting.

\section{Immunoblotting}

Proteins were resolved on SDS-PAGE and transferred to Immobilon-P membranes (Millipore). Membranes were blocked for $1 \mathrm{~h}$ in TBS containing 5\% BSA, followed by incubation with primary antibodies. After incubation with horseradish peroxidase-coupled secondary antibodies, immunoblots were developed using enhanced chemiluminescence (ECLPlus, GE Healthcare).

\section{Cell viability assay}

HEK293 stable cell lines expressing GFP control, L4 wild type, or $\Delta$ loop mutant were infected with lentiviruses encoding shRNA targeting RPL4 or scramble control. $48 \mathrm{~h}$ after infection, cells were trypsinized and plated at equal density to the 96-well plate. Cell viability was measured on day 3 using CCK-8 kit (Dojindo Molecular Technologies) according to the manufacturer's instructions.

\section{Ribosome profiling}

Sucrose solutions were prepared in polysome gradient buffer (10 mM Hepes, pH 7.4, $100 \mathrm{mM} \mathrm{KCl,} 5 \mathrm{mM} \mathrm{MgCl}_{2}, 100 \mu \mathrm{g} / \mathrm{ml}$ CHX, $5 \mathrm{mM}$ DTT, and $20 \mathrm{U} / \mathrm{ml}$ SUPERase_In (Ambion)). Sucrose density gradients $(15 \%-45 \%(\mathrm{wt} / \mathrm{vol}))$ were freshly made in SW41 ultracentrifuge tubes (Fisher) using a BioComp Gradient Master (BioComp) according to the manufacturer's instructions. Cells were plated to four $10-\mathrm{cm}$ dishes before ribosome profiling. HEK293 cells were first treated with CHX $(100 \mu \mathrm{M})$ or LTM (50 $\mu \mathrm{M}$ ) for $30 \mathrm{~min}$ at $37^{\circ} \mathrm{C}$ to freeze the translating ribosomes or initiating ribosomes, respectively. After ice-cold PBS solution wash, cells were then harvested by ice-cold polysome lysis buffer (10 mM Hepes, pH 7.4, $100 \mathrm{mM} \mathrm{KCl,} 5 \mathrm{mM} \mathrm{MgCl}, 100 \mu \mathrm{g} / \mathrm{ml} \mathrm{CHX}$, $5 \mathrm{mM}$ DTT, $20 \mathrm{U} / \mathrm{ml}$ SUPERase_In, and 2\% (vol/vol) Triton $\mathrm{X}-100)$. After centrifugation at $4{ }^{\circ} \mathrm{C}$ and $10000 \times g$ for $10 \mathrm{~min}$, approximately $650 \mu 1$ supernatant was loaded onto sucrose gradients, followed by centrifugation for $100 \mathrm{~min}$ at $38000 \mathrm{rpm}, 4{ }^{\circ} \mathrm{C}$, in an SW41 rotor. Separated samples were fractionated at $0.375 \mathrm{ml} /$ min by using a fractionation system (Isco) that continually monitored OD254 values. Fractions were collected into tubes at 1-min intervals. To convert the polysome into monosome, E. coli RNase
I (Ambion) was added into the pooled polysome samples (750 U per 100 A260 units) and incubated at $4{ }^{\circ} \mathrm{C}$ for $1 \mathrm{~h}$. SUPERase_In (50 U per $100 \mathrm{U}$ RNase I) was then added to stop digestion. Total RNA extraction was performed using TRIzol reagent.

\section{cDNA library construction of RPF}

Purified RNA samples were dephosphorylated in a $15 \mu 1$ reaction containing $1 \times \mathrm{T} 4$ polynucleotide kinase buffer, 10 U SUPERase_In, and 20 U T4 polynucleotide kinase (NEB). Dephosphorylation was carried out for $1 \mathrm{~h}$ at $37^{\circ} \mathrm{C}$, and the enzyme was then heat inactivated for $20 \mathrm{~min}$ at $65{ }^{\circ} \mathrm{C}$. Dephosphorylated samples were then mixed with 2 Novex TBE-Urea sample buffer (Invitrogen) and loaded on a Novex denaturing $15 \%$ polyacrylamide TBEurea gel (Invitrogen). The gel was stained with SYBR Gold (Invitrogen) to visualize the RNA fragments. Gel bands containing RNA species corresponding to $28 \mathrm{nt}$ were excised and physically disrupted by centrifugation through the holes of the tube. RNA fragments were dissolved by soaking overnight in gel elution buffer (300 mM NaOAc, pH 5.5, 1 mM EDTA, 0.1 U/ml SUPERase_In). The gel debris was removed using a Spin-X column (Corning) and RNA was purified by using ethanol precipitation. Purified RNA fragments were resuspended in $10 \mathrm{mM}$ Tris $(\mathrm{pH} 8)$ and denatured briefly at $65{ }^{\circ} \mathrm{C}$ for $30 \mathrm{~s}$. Poly-(A) tailing reaction was performed in a $8 \mu \mathrm{l}$ buffer with $1 \times$ poly-(A) polymerase, $1 \mathrm{mM}$ ATP, $0.75 \mathrm{U} /$ $\mu 1$ SUPERase_In, and 3 U E. coli poly-(A) polymerase (NEB). Tailing was carried out for $45 \mathrm{~min}$ at $37{ }^{\circ} \mathrm{C}$. For reverse transcription, the following oligos containing barcodes were synthesized:

MCA02, 5'-pCAGATCGTCGGACTGTAGAACTCT/idSp/CAAGCAGAAGACGGCATACGATTTTTTTTTTTTTTTTTTTTVN-3'; LGT03, 5'-pGTGATCGTCGGACTGTAGAACTCT/idSp/CAAGCAGAAGACGGCATACGATTTTTTTTTTTTTTTTTTTTVN-3'; YAG04, 5'-pTCGATCGTCGGACTGTAGAACTCT/idSp/CAAGCAGAAGACGGCATACGATTTTTTTTTTTTTTTTTTTTVN-3'; HTC05, 5'-pAGGATCGTCGGACTGTAGAACTCT/idSp/CAAGCAGAAGACGGCATACGATTTTTTTTTTTTTTTTTTTTVN-3'.

In brief, the tailed RNA product was mixed with $0.5 \mathrm{mM} \mathrm{dNTP}$ and $2.5 \mathrm{mM}$ synthesized primer and incubated at $65^{\circ} \mathrm{C}$ for $5 \mathrm{~min}$, followed by incubation on ice for $5 \mathrm{~min}$. The reaction mix was then added with $20 \mathrm{mM}$ Tris ( $\mathrm{pH} 8.4$ ), $50 \mathrm{mM} \mathrm{KCl}, 5 \mathrm{mM} \mathrm{MgCl}_{2}$, $10 \mathrm{mM}$ DTT, 40 U RNaseOUT, and 200 U SuperScript III (Invitrogen). Reverse transcription reaction was performed according to the manufacturer's instructions. RNA was eliminated from cDNA by adding $1.8 \mu \mathrm{l}$ of $1 \mathrm{M} \mathrm{NaOH}$ and incubating at $98^{\circ} \mathrm{C}$ for $20 \mathrm{~min}$. The reaction was then neutralized with $1.8 \mu \mathrm{l}$ of $1 \mathrm{M} \mathrm{HCl}$. Reverse transcription products were separated on a $10 \%$ polyacrylamide TBE-urea gel as described earlier. The extended first-strand product band was expected to be approximately $100 \mathrm{nt}$, and the corresponding region was excised. The cDNA was recovered by using DNA gel elution buffer ( $300 \mathrm{mM} \mathrm{NaCl}, 1 \mathrm{mM}$ EDTA). First-strand cDNA was circularized in $20 \mu \mathrm{l}$ of reaction containing $1 \times \mathrm{CircLi}$ -

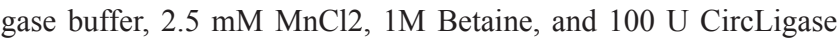
II (Epicentre). Circularization was performed at $60^{\circ} \mathrm{C}$ for $1 \mathrm{~h}$, and the reaction was heat inactivated at $80^{\circ} \mathrm{C}$ for $10 \mathrm{~min}$. Circular single-strand DNA was relinearized with $20 \mathrm{mM}$ Tris-acetate, $50 \mathrm{mM}$ potassium acetate, $10 \mathrm{mM}$ magnesium acetate, $1 \mathrm{mM}$ DTT, and 7.5 U APE 1 (NEB). The reaction was carried out at $37^{\circ} \mathrm{C}$ for $1 \mathrm{~h}$. The linearized single-strand DNA was separated on a Novex $10 \%$ polyacrylamide TBE-urea gel (Invitrogen) as described earlier. The expected 100-nt product bands were excised and recovered as 
described earlier.

\section{Deep sequencing}

Single-stranded template was amplified by PCR using the Phusion High-Fidelity enzyme (NEB) according to the manufacturer's instructions. The oligonucleotide primers qNTI200 (5'-CAAGCAGAAGACGGCATA-3') and qNTI201 (5'-AATGATACGGCGACCACCGACAGGTTCAGAGTTCTACAGTCCGACG-3') were used to create DNA suitable for sequencing, i.e., DNA with Illumina cluster generation sequences on each end and a sequencing primer binding site. The PCR contains $1 \times$ HF buffer, $0.2 \mathrm{mM}$ dNTP, $0.5 \mu \mathrm{M}$ oligonucleotide primers, and $0.5 \mathrm{U}$ Phusion polymerase. PCR was carried out with an initial 30 -s denaturation at $98^{\circ} \mathrm{C}$, followed by 12 cycles of 10 -s denaturation at $98^{\circ} \mathrm{C}, 20$-s annealing at $60{ }^{\circ} \mathrm{C}$, and $10 \mathrm{~s}$ extension at $72{ }^{\circ} \mathrm{C}$. PCR products were separated on a nondenaturing $8 \%$ polyacrylamide TBE gel as described earlier. Expected DNA at $120 \mathrm{bp}$ was excised and recovered as described earlier. After quantification by Agilent BioAnalyzer DNA 1000 assay, equal amount of barcoded samples were pooled into one sample. Approximately 3-5 pM mixed DNA samples were used for cluster generation followed by sequencing using sequencing primer 5'-CGACAGGTTCAGAGTTCTACAGTCCGACGATC-3' (Illumina Genome Analyzer 2 or HiSeq).

\section{Data analysis}

Barcoded reads were separated into individual data file for each sample according to the $5^{\prime}$ end 2-nt barcode sequences. Seven nucleotides were trimmed from the $3^{\prime}$ end of each 50-nt-long Illumina sequence read to remove adaptor sequences, and the poly (A) tails were removed from the $3^{\prime}$ end, allowing one mismatch. Reads between 25 and $35 \mathrm{nt}$ in length were mapped using Bowtie [31] to the sense strand of the longest Refseq transcripts for each human gene. One mismatch was allowed in all mappings; in cases of multiple mapping, mismatched positions were not used if a perfect match existed. Reads mapped more than 100 times were discarded to remove poly-A-derived reads. Finally, reads were counted at every position of individual transcripts by using the 13th nucleotide of the read for the P-site position.

To generate aggregation plots, the number of RPF reads aligned to each position of individual transcripts was first normalized by the read density within 50-150 codon window, which was considered as the representative translational level of the transcript. Only transcripts with CDS longer than 150 codons and with minimum read density of $25 \mathrm{rpkM}$ were used for aggregation plots. The read counts were then averaged across all transcripts for each position relative to the annotated start codon based on Refseq.

The framing of reads mapped to the 1st codon, 5th codon, or entire CDS was calculated relative to the annotated ORF based on Refseq. The length distribution was also calculated for reads mapped to the 1 st codon, the 5 th codon, or the entire CDS. Read density over the CDS (6-stop codon) relative to the initiation site (1-5 codon) was computed for each transcript. Only transcripts with minimum read density of $10 \mathrm{rpkM}$ over the CDS and minimum 5 reads at the initiation site were used to calculate the ratios.

For codon composition analysis, the annotated CDS from human gene was downloaded from UCSC table browser. The codon composition of the 1 st codon, 5th codon, and whole CDS were summarized based on the longest transcripts of human genes.

For GO term enrichment analysis, two groups of genes were collected based on the average read densities in the regions flank- ing the start codon and the 5th codon by the following criteria:

Group I: Density ${ }_{\text {Ist codon }}>1.2 \times$ Density $_{5 \text { th codon }}$

Group II: Density ${ }_{5 \text { th codon }}>1.2 \times$ Density $_{\text {sth codon }}$

The gene ontology analyses were conducted on the above two groups of genes using DAVID [32].

\section{Acknowledgments}

We thank members of the Qian laboratory for critical reading of the manuscript and Dr Jonathan Yewdell (NIAID, NIH) for providing anti-puromycin monoclonal antibody. B L is partly supported by the Genomics Scholar's Award from Center for Vertebrate Genomics at Cornell. This work was supported by grants from National Institutes of Health (1 DP2 OD006449-01), Ellison Medical Foundation (AG-NS-0605-09), and DOD Exploration-Hypothesis Development Award (W81XWH-11-1-0236) to S-B Q.

\section{References}

1 Buchan JR, Stansfield I. Halting a cellular production line: responses to ribosomal pausing during translation. Biol Cell 2007; 99:475-487.

2 Kertesz M, Wan Y, Mazor E, et al. Genome-wide measurement of RNA secondary structure in yeast. Nature 2010; 467:103-107.

3 Plotkin JB, Kudla G. Synonymous but not the same: the causes and consequences of codon bias. Nat Rev Genet 2011; 12:32-42.

4 Kramer G, Boehringer D, Ban N, Bukau B. The ribosome as a platform for co-translational processing, folding and targeting of newly synthesized proteins. Nat Struct Mol Biol 2009; 16:589-597.

5 Farabaugh PJ. Translational frameshifting: implications for the mechanism of translational frame maintenance. Prog $\mathrm{Nu}$ cleic Acid Res Mol Biol 2000; 64:131-170.

6 Doma MK, Parker R. Endonucleolytic cleavage of eukaryotic mRNAs with stalls in translation elongation. Nature 2006; 440:561-564.

7 Komar AA. A pause for thought along the co-translational folding pathway. Trends Biochem Sci 2009; 34:16-24.

8 Liu B, Han Y, Qian SB. Cotranslational response to proteotoxic stress by elongation pausing of ribosomes. Mol Cell 2013; 49:453-463.

9 Shalgi R, Hurt JA, Krykbaeva I, Taipale M, Lindquist S, Burge CB. Widespread regulation of translation by elongation pausing in heat shock. Mol Cell 2013; 49:439-452.

10 Wolin SL, Walter P. Ribosome pausing and stacking during translation of a eukaryotic mRNA. EMBO J 1988; 7:35593569.

11 Ingolia NT, Ghaemmaghami S, Newman JR, Weissman JS. Genome-wide analysis in vivo of translation with nucleotide resolution using ribosome profiling. Science 2009; 324:218223.

12 Guo H, Ingolia NT, Weissman JS, Bartel DP. Mammalian microRNAs predominantly act to decrease target mRNA levels. Nature 2010; 466:835-840.

13 Brar GA, Yassour M, Friedman N, Regev A, Ingolia NT, Weissman JS. High-resolution view of the yeast meiotic pro- 
gram revealed by ribosome profiling. Science 2012; 335:552557.

14 Oh E, Becker AH, Sandikci A, et al. Selective ribosome profiling reveals the cotranslational chaperone action of trigger factor in vivo. Cell 2011; 147:1295-1308.

15 Li GW, Oh E, Weissman JS. The anti-Shine-Dalgarno sequence drives translational pausing and codon choice in bacteria. Nature 2012; 484:538-541.

16 Bazzini AA, Lee MT, Giraldez AJ. Ribosome profiling shows that miR-430 reduces translation before causing mRNA decay in zebrafish. Science 2012; 336:233-237.

17 Stadler M, Artiles K, Pak J, Fire A. Contributions of mRNA abundance, ribosome loading, and post- or peri-translational effects to temporal repression of C. elegans heterochronic miRNA targets. Genome Res 2012; 22:2418-2426.

18 Gerashchenko MV, Lobanov AV, Gladyshev VN. Genome-wide ribosome profiling reveals complex translational regulation in response to oxidative stress. Proc Natl Acad Sci USA 2012; 109:17394-17399.

19 Lee S, Liu B, Huang SX, Shen B, Qian SB. Global mapping of translation initiation sites in mammalian cells at single-nucleotide resolution. Proc Natl Acad Sci USA 2012; 109:E2424-E2432.

20 Guydosh NR, Green R. Dom34 rescues ribosomes in 3' untranslated regions. Cell 2014; 156:950-962.

21 O'Connor PB, Li GW, Weissman JS, Atkins JF, Baranov PV. rRNA:mRNA pairing alters the length and the symmetry of mRNA-protected fragments in ribosome profiling experiments. Bioinformatics 2013; 29:1488-1491.

22 Schneider-Poetsch T, Ju J, Eyler DE, et al. Inhibition of eukaryotic translation elongation by cycloheximide and lactimidomycin. Nat Chem Biol 2010; 6:209-217.
23 Rooijers K, Loayza-Puch F, Nijtmans LG, Agami R. Ribosome profiling reveals features of normal and disease-associated mitochondrial translation. Nat Commun 2013; 4:2886.

24 Wilson DN, Beckmann R. The ribosomal tunnel as a functional environment for nascent polypeptide folding and translational stalling. Curr Opin Struct Biol 2011; 21:274-282.

25 Klinge S, Voigts-Hoffmann F, Leibundgut M, Arpagaus S, Ban N. Crystal structure of the eukaryotic 60S ribosomal subunit in complex with initiation factor 6. Science 2011; 334:941-948.

26 Starosta AL, Karpenko VV, Shishkina AV, et al. Interplay between the ribosomal tunnel, nascent chain, and macrolides influences drug inhibition. Chem Biol 2010; 17:504-514.

27 Heurgue-Hamard V, Mora L, Guarneros G, Buckingham RH. The growth defect in Escherichia coli deficient in peptidyl-tRNA hydrolase is due to starvation for Lys-tRNA(Lys). EMBO $J$ 1996; 15:2826-2833.

28 Petrov A, Kornberg G, O'Leary S, Tsai A, Uemura S, Puglisi JD. Dynamics of the translational machinery. Curr Opin Struct Biol 2011; 21:137-145.

29 Tenson T, Ehrenberg M. Regulatory nascent peptides in the ribosomal tunnel. Cell 2002; 108:591-594.

30 Zhang Y, Wolfle T, Rospert S. Interaction of nascent chains with the ribosomal tunnel proteins Rp14, Rp117, and Rp139 of Saccharomyces cerevisiae. J Biol Chem 2013; 288:3369733707.

31 Langmead B, Trapnell C, Pop M, Salzberg SL. Ultrafast and memory-efficient alignment of short DNA sequences to the human genome. Genome Biol 2009; 10:R25.

32 Huang da W, Sherman BT, Lempicki RA. Systematic and integrative analysis of large gene lists using DAVID bioinformatics resources. Nat Protoc 2009; 4:44-57.

(Supplementary information is linked to the online version of the paper on the Cell Research website.) 